\section{Characterization of a new ultrasound device for clinical diagnosis}

\section{Valentina Tavoni \\ Department of Physics and Vascular Diseases Center, University of Ferrara, Ferrara, Italy}

\section{Background}

During recent years, it has been recognized that a poor or anomalous blood flow in the internal jugular veins (IJVs) can be related to brain drainage problems. One of the most interesting parameters to study in respect to the functionality of the IJVs is the jugular venous pulse (JVP) trace. In recent years, my research group demonstrated how to obtain the JVP trace with a B-mode ultrasound (US) examination, measuring the changes of cross sectional area of the veins over time. ${ }^{1}$ Moreover, it is possible to quantify the blood flow inside the IJVs measuring in Doppler mode the time average velocity of the blood cells.

Despite the fact that the US technology is commonly used in many diagnostic fields, the measures could be strongly affected by human error due to the operator dependence on the US device. To reduce this kind of error, the University of Florence (Italy) developed a new experimental device: the US Advanced Open Platform (ULA-OP). ${ }^{2}$

\section{Methods}

In this work we characterized the performances of the ULA-OP during an haemodynamic study. In particular we compared the behavior of this device with a standard US device in the two main modalities used during a cardiovascular examination. We started from a previous paper about the analysis of the Doppler mode in vitro, performed using a static phantom and a continuous flow pump. ${ }^{3}$ Then we completed the study analysing the ULA-OP performances in B-mode studying in vivo the IJVs of 8 healthy volunteers.

\section{Results}

The results showed that in Doppler mode the ULA-OP system is more accurate but less precise than the standard one. Regarding the B-mode the performances of the two devices are very similar, even if the image quality of the ULA-OP is worse. However, the high programmability of the ULA-OP, one of the main innovations of this device, could compensate for all these defects.

\section{Conclusions}

Once the necessaries adjustments have been implemented, the ULA-OP platform could become very useful for cardiovascular diagnostics and in general for many other clinical fields.
Correspondence: Valentina Tavoni, Department of Physics and Vascular Diseases Center, University of Ferrara, Ferrara, Italy. E-mail: tvnvnt@unife.it

Key words: Neurovascular diseases; meeting.

Conference presentation: $9^{\text {th }}$ Annual Meeting of the International Society of Neurovascular Disease (ISNVD), May $30^{\text {th }}-31^{\text {st }}, 2019$, Ferrara, Italy.

This work is licensed under a Creative Commons Attribution 4.0 License (by-nc 4.0).

(C) Copyright: the Author(s), 2019

Licensee PAGEPress, Italy

Veins and Lymphatics 2019; 8:8434

doi:10.4081/vl.2019.8434

\section{References}

1. Sisini F, Tessari M, Gadda G, et al. An ultrasonographic Technique to assess the jugular venous pulse: a proof of concept. Ultrasound Med Bio 2015;41: 1334-41.

2. Tortoli P, Bassi L, Boni E, et al. ULAOP: an advanced open platform for ultrasound research. IEEE Trans Ultrason Ferroelectr Freq Control 2009;56:2207-16.

3. Tavoni V, Sisini F, Di Domenico G, et al. Conventional echo color Doppler versus ULA-OP in the assessment of venous flow model. Veins and Lymphatics 2017;6:6841. 